\title{
Low-Exponent RSA with Related Messages
}

\author{
Don Coppersmith ${ }^{\star}$ Matthew Franklin $^{\star \star}$ Jacques Patarin $^{\star \star \star}$ Michael Reiter $^{\dagger}$
}

\begin{abstract}
In this paper we present a new class of attacks against RSA with low encrypting exponent. The attacks enable the recovery of plaintext messages from their ciphertexts and a known polynomial relationship among the messages, provided that the ciphertexts were created using the same RSA public key with low encrypting exponent.
\end{abstract}

\section{Introduction}

In this paper we present a new class of attacks against RSA [8] with low encrypting exponent. The attacks enable the recovery of plaintext messages from their ciphertexts and a known polynomial relationship among the messages, provided that the ciphertexts were created using the same RSA public key with low encrypting exponent. Our attacks differ from the low-exponent attacks described by Moore [6] and Hastad [5] and the common modulus attack identified by Simmons [10], which pertain only to ciphertexts encrypted under different public keys.

Given encryptions of $k$ messages under the same RSA public key with exponent $e$, together with knowledge of a polynomial relation of degree $\delta$ among the messages, the goal of the attacks is to recover all messages. Our results were influenced by an attack presented by Franklin and Reiter [4] for the case $k=2, e=3, \delta=1$. Starting with this case, we generalize the exponent $e$ in Section 2, the degree $\delta$ in Section 3, and the number of messages $k$ in Section 4. Implications of the attack are considered in Section 5 .

\section{Generalizing the exponent $e$}

Suppose we have two messages $m_{1}$ and $m_{2}$ related by a known affine relation

$$
m_{2}=\alpha m_{1}+\beta \text {. }
$$

Suppose further that the messages are encrypted under RSA with an exponent of 3 using a single public modulus $N$.

$$
c_{i}=m_{i}^{3} \bmod N, \quad i=1,2
$$

\footnotetext{
* IBM Research, Yorktown Heights, NY, USA; copper@uatson. ibm.com

* AT\&T Research, Murray Hill, NJ, USA; franklin@research.att.com

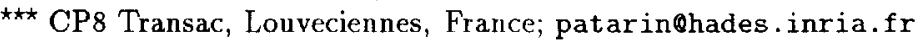

${ }^{\dagger}$ AT\&T Research, Murray Hill, NJ, USA; reiter@research.att.com

U. Maurer (Ed.): Advances in Cryptology - EUROCRYPT '96, LNCS 1070, pp. 1-9, 1996.

(c) Springer-Verlag Berlin Heidelberg 1996
} 
Then from

$$
c_{1}, c_{2}, \alpha, \beta, N
$$

we can calculate the secret messages $m_{i}$ algebraically as follows:

$$
\frac{\beta\left(c_{2}+2 \alpha^{3} c_{1}-\beta^{3}\right)}{\alpha\left(c_{2}-\alpha^{3} c_{1}+2 \beta^{3}\right)}=\frac{3 \alpha^{3} \beta m_{1}^{3}+3 \alpha^{2} \beta^{2} m_{1}^{2}+3 \alpha \beta^{3} m_{1}}{3 \alpha^{3} \beta m_{1}^{2}+3 \alpha^{2} \beta^{2} m_{1}+3 \alpha \beta^{3}}=m_{1} \bmod N \text {. }
$$

The algebra is more transparent if we assume (without loss of generality) that $\alpha=\beta=1$.

$$
\frac{(m+1)^{3}+2 m^{3}-1}{(m+1)^{3}-m^{3}+2}=\frac{3 m^{3}+3 m^{2}+3 m}{3 m^{2}+3 m+3}=m \bmod N .
$$

So if the RSA exponent is $e=3$ and we have $k=2$ messages, satisfying a known polynomial relation of degree $\delta=1$, we can recover the messages $m_{i}$ algebraically from the ciphertexts and the coefficients of the polynomial relation.

When $e=5$, setting $c_{1}=m^{5} \bmod N$ and $c_{2}=(m+1)^{5} \bmod N$, we can find

$$
\begin{aligned}
P(m) & =c_{2}^{3}-3 c_{1} c_{2}^{2}+3 c_{1}^{2} c_{2}-c_{1}^{3}+37 c_{2}^{2}+176 c_{1} c_{2}+37 c_{1}^{2}+73 c_{2}-73 c_{1}+14 \\
m P(m) & =2 c_{2}^{3}-1 c_{1} c_{2}^{2}-4 c_{1}^{2} c_{2}+3 c_{1}^{3}+14 c_{2}^{2}-88 c_{1} c_{2}-51 c_{1}^{2}-9 c_{2}+64 c_{1}-7 \\
m & =\frac{m P(m)}{P(m)}
\end{aligned}
$$

For an arbitrary exponent $e$ in the case of $k=2$ messages subject to a linear relation, it will always be possible to write down an equation analogous to (1). Specifically, there will exist polynomials $P(m)$ and $Q(m)$ such that each can be expressed as rational polynomials in $m^{e}$ and $(m+1)^{e}$, and such that $Q(m)=m P(m)$. Already for $e=5$, however, this is fairly complicated. As $e$ grows, this explicit expression of $m$ as a ratio of two polynomials in $c_{1}$ and $c_{2}$ requires $\Theta\left(e^{2}\right)$ coefficients, and it is not immediately obvious how to calculate these coefficients efficiently.

Fortunately there is an easier method. Let $z$ denote the unknown message $m$. Then $z$ satisfies the following two polynomial relations:

$$
\begin{aligned}
z^{5}-c_{1} & =0 \bmod N \\
(z+1)^{5}-c_{2} & =0 \bmod N .
\end{aligned}
$$

where the $c_{i}$ are treated as known constants. Apply the Euclidean algorithm to find the greatest common divisor of these two univariate polynomials over the ring $\mathbf{Z} / N$ :

$$
\operatorname{gcd}\left(z^{5}-c_{1},(z+1)^{5}-c_{2}\right) \in \mathbf{Z} / N[z]
$$

This should yield the linear polynomial $z-m$ (except possibly in rare cases ${ }^{1}$ ).

${ }^{1}$ We do not fully understand the cases $m=\left(1-\omega^{j}\right) /(\omega-1) \in \mathrm{Z} / p, 2 \leq j \leq e-1$, where $p$ is a prime factor of $N$ and $\omega$ is a primitive eth root of 1 in some extension of $\mathbf{Z} / p$. This condition seems to imply that $e \mid p-1$, in contradiction to the RSA requirement $\operatorname{gcd}(e, p-1)=1$, but work needs to be done to verify this. Other than these at most $(e-1)(e-2)$ possible exceptions, the gcd will in fact be linear. 
The attack applies for any value of $e$, but is limited by the cost of computing the gcd of two polynomials of degree e. A straightforward implementation of Euclid's algorithm takes $O\left(e^{2}\right)$ operations in the ring $Z / N$. More sophisticated techniques can be used to compute the gcd in $O\left(e \log ^{2} e\right)$ time [12]. Using these methods, the attack may be practical for all exponents of length up to around 32 bits. For example, the attack will be very efficient against $e=2^{16}+1$, a popular choice in many applications.

\section{Generalizing the degree $\delta$ of the polynomial}

One generalization is immediate. Suppose we have two messages $m_{1}, m_{2}$ satisfying a known polynomial relation of the form

$$
m_{2}=p\left(m_{1}\right), \quad \operatorname{deg}(p)=\delta,
$$

and we know $p$ and the two ciphertexts $c_{i}$. Then as before, the two equations

$$
\begin{aligned}
z^{e}-c_{1} & =0 \bmod N \\
(p(z))^{e}-c_{2} & =0 \bmod N
\end{aligned}
$$

are both satisfied by $z=m_{1} \bmod N$ so that

$$
\operatorname{gcd}\left(z^{e}-c_{1},(p(z))^{e}-c_{2}\right) \in \mathbf{Z} / N[z]
$$

will be divisible by $z-m_{1}$, and except in rare cases we will have

$$
\operatorname{gcd}\left(z^{e}-c_{1},(p(z))^{e}-c_{2}\right)=z-m_{1}
$$

(One of the exceptional cases, in which this procedure fails, is when $p(z)$ is of the form $p(z)=z^{h} q\left(z^{e}\right)$, because then the ciphertext $c_{2}$ is easily derived from the ciphertext $c_{1}$, namely

$$
c_{2}=c_{1}^{h}\left(q\left(c_{1}\right)\right)^{e},
$$

and we gain no new information.)

What if $m_{1}$ and $m_{2}$ satisfy an implicit polynomial relation?

$$
p\left(m_{1}, m_{2}\right)=0 \bmod N
$$

In this case we have three polynomials relating two unknowns $\bmod N$ :

$$
\begin{aligned}
& P_{1}=p\left(m_{1}, m_{2}\right)=0 \bmod N \\
& P_{2}=m_{1}^{e}-c_{1}=0 \bmod N \\
& P_{3}=m_{2}^{e}-c_{2}=0 \bmod N
\end{aligned}
$$

Now we need another algebraic tool: the resultant. The resultant of two multivariate polynomials, with respect to one of their variables $x$, is a third multivariate polynomial, in all the variables except the special variable $x$. For any setting of all the variables (including $x$ ) for which the two input polynomials are 
simultarleously 0 , the resultant is also 0 . This gives us a means of eliminating $x$ from a system of equations.

In the example above, substitute $x$ and $y$ for the unknowns $m_{1}$ and $m_{2}$, respectively. We can take the resultant of $P_{1}(x, y)$ and $P_{2}(x)$ with respect to the variable $x$, over the ring $Z / N$. This will yield a polynomial $P_{4}$ in $y$, whose degree is at most $\delta e$ (the product of the total degrees of the original polynomials). Then we can take the ged of this new polynomial $P_{4}$ with $P_{3}$ (as univariate polynomials in $y$ over $\mathbf{Z} / N$ ) to yield (hopefully) the linear polynomial $y-m_{2}$. Substitute this value of $m_{2}$ which we have discovered, back into $P_{1}$, to find a polynomial in $x$ alone, which we can combine with $P_{2}$ by the gcd to find the correct value of $x=m_{1}$. The complexity of this attack is dominated by the conputation of the resultant, i.e., the determinant of a $(\delta+e) \times(\delta+e)$ matrix whose elements are univariate polynomials of degree $\delta$. This can naively be done in $O\left((\delta+e)^{3} \delta^{2}\right)$ operations.

The two operations, resultant and gcd, can be combined under the general heading of "Groebner basis." Indeed, fixing the coefficients of $p$ and the ciphertexts $c_{i}$ as constants, and computing the Gruebner basis of $P_{1}(x, y), P_{2}(x), P_{3}(y)$, as polynomials over $\mathbf{Z} / N$, will generally produce the result $\left[\left(x-m_{1}\right),\left(y-m_{2}\right)\right]$. In this paper, however, we work explicitly with the resultant and ged, in order to better estimate the complexity of the attacks.

\section{Generalizing the number of messages $k$}

\subsection{Arbitrary polynomial relationship among messages}

Suppose we have $k$ messages $m_{1}, \ldots, m_{k}$, related by a polynomial $p\left(m_{1}, \ldots, m_{k}\right)$, and that we know the ciphertexts $c_{i}=m_{i}^{e} \bmod N$ and the coefficients of the polynomial $p$. As before, substitute variables $x_{i}$ for the unknown messages $m_{i}$, and obtain the $k+1$ polynomials

$$
\begin{aligned}
& P_{0}\left(x_{1}, \ldots, x_{k}\right)=p\left(x_{1}, \ldots, x_{k}\right)=0 \bmod N \\
& P_{1}\left(x_{1}\right)=x_{1}^{e}-c_{1}=0 \bmod N \\
& P_{2}\left(x_{2}\right)=x_{2}^{e}-c_{2}=0 \bmod N \\
& P_{i}\left(\ddot{x}_{i}\right)=x_{i}^{e}-c_{i}=0 \bmod N \\
& P_{k}\left(x_{k}\right)=x_{k}^{e}-c_{k}=0 \bmod N
\end{aligned}
$$

which must be simultaneously satisfied. We can just compute

$$
\text { Groebner }\left(\left[P_{0}, P_{1}, \ldots, P_{k}\right]\right)
$$

and generally obtain the answer

$$
\left[x_{1}-m_{1}, \ldots, x_{k}-m_{k}\right] .
$$

Or, more explicitly, we can set

$$
Q_{0}\left(x_{1}, \ldots, x_{k i}\right)=P_{0}
$$


and iteratively evaluate

$$
Q_{i}\left(x_{i+1}, \ldots, x_{k}\right)=\text { Resultant }_{x_{i}}\left(Q_{i-1}, P_{i}\right)
$$

until we find

$$
Q_{k-1}\left(x_{k}\right)
$$

Then evaluating

$$
\operatorname{gcd}\left(Q_{k-1}\left(x_{k}\right), P_{k}\left(x_{k}\right)\right)
$$

we hope to find the linear polynomial $\left(x_{k}-m_{k}\right)$, from which we discover $m_{k}$. Finally, repeatedly back substitute:

$$
\operatorname{gcd}\left(P_{i}\left(x_{i}\right), Q_{i-1}\left(x_{i}, m_{i+1}, m_{i+2}, \ldots, m_{k}\right)\right)=\left(x_{i}-m_{i}\right),
$$

as $i$ goes from $k-1$ to 1 , to find all the messages $m_{i}$.

The complexity of this general attack is dominated by the computation of the resultants $Q_{1}, \ldots, Q_{k-1}$. Each $Q_{i}$ is a polynomial of total degree $e^{i} \delta$, in $k-i$ variables. The number of monomial terms in each $Q_{i}$ is potentially as large as $\Theta\left(\delta^{k / 2} e^{k^{2} / 4}\right)$. Thus, this attack may require $\delta^{\Theta(k)} e^{\Theta\left(k^{2}\right)}$ operations over $\mathbf{Z} / N$.

\subsection{A special case: linearly related messages}

As will be seen in Section 5.2, a special case of interest is that in which $P_{0}=p$ is linear, say

$$
p\left(x_{1}, \ldots, x_{k}\right)=x_{1}+x_{2}+\ldots+x_{k}-w
$$

with $w$ some known constant. The special form of $p$ allows us to make the computations more efficient. In addition, the success of the attack differs depending on whether $w$ is zero or nonzero.

Inhomogeneous case. In the inhomogeneous case $w \neq 0$, the attack proceeds as follows. Introduce unknowns

$$
y_{i}=x_{1}+x_{2}+\ldots+x_{i}=w-x_{k}-x_{k-1}-\ldots-x_{i+1}
$$

satisfying

$$
\begin{aligned}
& y_{i-1}=y_{i}-x_{i} \\
& y_{i+1}=y_{i}+x_{i+1}
\end{aligned}
$$

Set $h=\lfloor k / 2\rfloor$. Introduce polynomials:

$$
\begin{aligned}
R_{1}\left(y_{1}\right) & =P_{1}\left(y_{1}\right) \\
R_{i}\left(y_{i}\right) & =\operatorname{Resultant}_{x_{1}}\left(R_{i-1}\left(y_{i}-x_{i}\right), P_{i}\left(x_{i}\right)\right), \quad i=1,2, \ldots, h \\
S_{k-1}\left(y_{k-1}\right) & =P_{k}\left(w-y_{k-1}\right) \\
S_{i}\left(y_{i}\right) & =\operatorname{Resultant}_{x_{1}}\left(S_{i+1}\left(y_{i}+x_{i+1}\right), P_{i+1}\left(x_{i+1}\right)\right), \quad i=k-2, k-3, \ldots, h
\end{aligned}
$$


Both $R_{h}\left(y_{h}\right)$ and $S_{h}\left(y_{h}\right)$ are univariate polynomials in $y_{h}$. Their gcd will hopefully be the linear polynomial

$$
y_{h}-\left(m_{1}+m_{2}+\ldots+m_{h}\right)
$$

and we can proceed from there by divide-and-conquer.

A shortcut to computing each $R_{i}\left(y_{i}\right)$ is to evaluate $R_{i-1}\left(y_{i}-x_{i}\right)$ by Horner's rule, replacing each occurrence of $x_{i}^{e}$ by $c_{i}$. The complexity of the attack is dominated by the complexity of computing $\operatorname{gcd}\left(R_{h}\left(y_{h}\right), S_{h}\left(y_{h}\right)\right)$. This is $O\left(e^{k / 2} k^{2}\right)$ since the degrees of $R_{h}$ and $S_{h}$ are both $\Theta\left(e^{k / 2}\right)$.

Homogeneous case. A difficulty arises in the homogeneous case $w=0$. Because $P_{0}$ is homogeneous, and all the other polynomials are of the form $x^{e}-$ $c$, given any solution $\left(x_{1}, x_{2}, \ldots, x_{k}\right)$ and any $e$ th root of unity $\phi$, the tuple $\left(\phi x_{1}, \phi x_{2}, \ldots, \phi x_{k}\right)$ is also a solution of all the $P_{i}$. Thus the attacker cannot solve for the individual $x_{i}$; the most it can hope for is to be able to solve for all homogeneous polynomials of degree $e$ in the $x_{i}$. In particular, when the attacker attempts to compute $y_{h}$, the gcd yields at best something like

$$
y_{h}^{e}-\left(m_{1}+m_{2}+\ldots+m_{h}\right)^{e}
$$

rather than the desired linear polynomial. This is an inherent difficulty in our approach, and has a bearing on the application described in Section 5.2.

However, there are occasions when the attack succeeds even in the homogeneous case. For example, suppose

$$
\begin{aligned}
& m_{1}=\left(m+\lambda_{1} \beta\right) \bmod N \\
& m_{2}=\left(m+\lambda_{2} \beta\right) \bmod N \\
& m_{3}=\left(m+\lambda_{3} \beta\right) \bmod N
\end{aligned}
$$

where $\lambda_{1}, \lambda_{2}, \lambda_{3}$ are known, while $m, \beta$ are unknown, and $e=3$. Further suppose that $\beta$ is known to satisfy $\beta^{3}<N$. This is a homogeneous case, because the three plaintexts are known to satisfy the linear relation

$$
\left(\lambda_{3}-\lambda_{2}\right) m_{1}+\left(\lambda_{1}-\lambda_{3}\right) m_{2}+\left(\lambda_{2}-\lambda_{1}\right) m_{3}=0 \bmod N
$$

From the three ciphertexts $c_{i}=m_{i}^{3} \bmod N$ and the three coefficients $\lambda_{1}, \lambda_{2}, \lambda_{3}$, it is possible to solve for $B=\beta^{3} \bmod N$ : e.g., $B-\beta^{3}=\operatorname{gcd}(f, g)$, where

$$
\begin{aligned}
& f=\text { Resultant }_{m}\left(\left(m+\lambda_{1} \beta\right)^{3}-c_{1},\left(m+\lambda_{2} \beta\right)^{3}-c_{2}\right) \\
& g=\text { Resultant }_{m}\left(\left(m+\lambda_{1} \beta\right)^{3}-c_{1},\left(m+\lambda_{3} \beta\right)^{3}-c_{3}\right)
\end{aligned}
$$

Then $\beta$ can be recovered by computing the real cube root of $B$ (i.e., without modular reduction), from which $m$ can be recovered using previous techniques. 


\section{Implications}

Due to the widespread popularity of RSA with low encrypting exponent, our attacks potentially have implications to the security of a wide range of current and future cryptographic protocols. In this section we show how our attacks reveal vulnerabilities in two protocols.

\subsection{The TMN protocol}

In [13], Tatebayashi, Matsuzaki and Newman proposed a key distribution protocol. In this protocol, a passive eavesdropper sees $r_{1}^{e} \bmod N, r_{2}^{e} \bmod N$, and $r_{1}+r_{2} \bmod N$ exchanged among the protocol participants, where $e=3$ and $r_{1}, r_{2}$ are randomly generated values. The techniques of the previous sections enable a passive eavesdropper to learn the shared session key $r_{2}$ distributed in the protocol.

Simmons [11] previously found an active attack on this scheme (requiring two conspirators), for which three counter measures were suggested in [13]. The first two countermeasures -incorporating structure into $r_{1}$ and $r_{2}$, and prepending timestamps to $r_{1}$ and $r_{2}$-do not prevent our passive attack. The third, which assumes a shared secret key between the server and each party, appears to withstand our attack. Park et. al. [7] exploited the use of $e=3$ in TMN to show that after the same two parties exchange a session key three times, each has enough information to impersonate the other in future protocol executions. In contrast, our attack enables any eavesdropper to recover the session key exchanged in any run of the protocol. Nevertheless, our attack does not immediately apply to the fix proposed in [7].

\subsection{Verifiable signature sharing}

In [3], Franklin and Reiter presented a scheme to efficiently share an RSA signature of a known message among $n \geq 5 t+1$ servers so that the servers could verify the signature relation, despite the malicious misbehavior of up to $t$ of the servers. As part of this protocol, the holder of the signature shares the signature using Shamir's secret sharing scheme [9], i.e., by choosing at random a univariate polynomial

$$
B(x)=\sum_{j=0}^{t} b_{j} x^{j}
$$

over $\mathbf{Z} / N$ such that $b_{0}$ is the secret signature, and privately sending the share $B(i)$ to the $i$-th server for $1 \leq i \leq n$. The intention is to ensure that a subset of $t+1$ servers can use their shares to recover $B$ (by Lagrange interpolation), but to prevent $t$ or fewer malicious servers from doing so. However, the holder of the signature also publishes the RSA encryption of each share under the same RSA public key with exponent $e=3$, i.e., $\left\{B(i)^{e} \bmod N\right\}_{1 \leq i \leq n}$.

The techniques of this paper enable one server $S_{i_{0}}$, knowing its share $B\left(i_{0}\right)$ and the ciphertexts $B\left(i_{1}\right)^{e} \bmod N, \ldots, B\left(i_{k}\right)^{e} \bmod N$ of at least $k=t+1$ other 
shares (i.e., $i_{0} \neq i_{j}$ for any $1 \leq j \leq k$ ), to compute the secret polynomial $B$. Specifically, because the shares $B\left(i_{0}\right), \ldots, B\left(i_{k}\right)$ are linear combinations of the unknowns $b_{j}$ with known coefficients, $S_{i_{0}}$ can compute a linear relation that holds among the shares:

$$
\sum_{j=0}^{k} p_{j} B\left(i_{j}\right)=0 \bmod N .
$$

Since server $S_{i_{0}}$ knows $p_{0} B\left(i_{0}\right)$, which is nonzero with high probability, it can learn an inhomogeneous linear relation among the other $k$ terms $\left\{p_{j} B\left(i_{j}\right)\right\}_{1 \leq j \leq k}$ :

$$
\sum_{j=1}^{k} p_{j} B\left(i_{j}\right)=-p_{0} B\left(i_{0}\right) \bmod N
$$

Using this information, and the easily computable ciphertext $p_{j}^{e} B\left(i_{j}\right)^{e} \bmod N$ of each term, it can use the techniques described before to recover each term $p_{j} B\left(i_{j}\right)$ and hence $B\left(i_{j}\right)$. Using Lagrange interpolation, it can then recover the secret polynomial $B$ and the signature $b_{0}$.

It is interesting to note that this attack fails for a passive eavesdropper that is not one of the $n$ servers. Such an eavesdropper sees only the published RSA encryptions of each share, i.e., $\left\{B(i)^{e} \bmod N\right\}_{1 \leq i \leq n}$. The eavesdropper can again find a linear equation of the form (2) among any $k+1$ of the shares. However, since this equation is homogeneous, it can recover only homogeneous polynomials of degree $e$ in the terms $p_{j} B\left(i_{j}\right)$ (see Section 4.2).

\section{Conclusion}

We have identified a new class attacks against RSA with low encrypting exponent, which exploit known polynomial relationships among the encrypted messages. This can lead to weaknesses in protocols for which such relationships can be inferred. When the relationships are essential to the correctness of a protocol, as in the case of Section 5.2, the only repair seems to be increasing the size of the encrypting exponent. If the polynomial relationships are not essential, then another repair might be to transform the plaintexts so that those relationships no longer hold. Possible transformations are applying a public permutation, such as DES with a fixed key, or padding the plaintext with random bits (though this may not always suffice; see [2]). Such transformations are discussed, e.g., in [1].

\section{References}

1. M. Bellare and P. Rogaway. Optimal asymmetric encryption. In Advances in Cryptology-EUROCRYPT '94 (Lecture Notes in Computer Science 950), A. De Santis, Ed. 1995, pp. 92-111, Springer-Verlag.

2. D. Coppersmith. Finding a small root of a univariate modular equation. In $A d$ vances in Cryptology - EUROCRYPT' $96, \mathrm{U}$. Maurer, Ed. 1996, Springer-Verlag. 
3. M. K. Franklin and M. K. Reiter. Verifiable signature sharing. In Advances in Cryptology-EUROCRYPT '95 (Lecture Notes in Computer Science 921), L. C. Guillou and J. Quisquater, Eds. 1995, pp. 50-63, Springer-Verlag.

4. M. K. Franklin and M. K. Reiter. A linear protocol failure for RSA with exponent three. Presented at the CRYPTO '95 Rump Session, Aug. 1995.

5. J. Hastad. Solving simultaneous modular equations of low degree. SIAM Journal of Computing 17:336-341, 1988.

6. J. H. Moore. Protocol failures in cryptosystems. Proceedings of the IEEE 76(5), May 1988.

7. C. Park, K. Kurosawa, T. Okamoto, and S. Tsujii. On key distribution and authentication in mobile radio networks. In Advances in Cryptology-EUROCRYPT '93 (Lecture Notes in Computer Science 765), T. Helleseth, Ed. 1994, pp. 461-465, Springer-Verlag.

8. R. L. Rivest, A. Shamir and L. Adleman. A method for obtaining digital signatures and public-key cryptosystcms. Communications of the ACM 21(2):120-126, Feb. 1978.

9. A. Shamir. How to share a secret. Communications of the ACM 22(11):612-613, Nov. 1979.

10. G. Simmons. A "weak" privacy protocol using the RSA cryptoalgorithm. Cryptologia 7:180-182, 1983.

11. G. Simmons. Proof of soundness (integrity) of cryptographic protocols. Journal of Cryptology 7:69-77, 1994.

12. V. Strassen. The computational complexity of continued fractions. SIAM Journal of Computing 12(1):1-27, 1983.

13. M. Tatebayashi and N. Matsuzakai and D. B. Newman. Key distribution protocol for digital mobile communication systems. In Advances in Cryptology-CRYPTO '89 (Lecture Notes in Computer Science 435), G. Brassard, Ed. 1990, pp. 324-333, Springer-Verlag. 